\title{
Computer Aided Screening Of Cervical Cancer From Pap Smear Microscopic Images
}

\author{
Brindha $\mathrm{D}^{1 *}$, Gauthaam $\mathrm{M}^{3}$ \\ \{*brn.bme@psgtech.ac.in\}, gauthaam.shibu@gmail.com \\ ${ }^{1}$ Department of Biomedical Engineering, PSG College of Technology, Coimbatore, India, ${ }^{3}$ Associate \\ Director ,Crowd ANALYTIX, Bangalore, India
}

\begin{abstract}
Cervical Cancer is globally ranked as the second among the type of cancers found in females. If diagnosed at early stage cervical cancer can be preventable type of cancer. Precancerous cervical cell changes generally do not cause symptoms, and hence it has prompted for an extensive research. An automated system for screening cervical cancer from pap-smear images have been developed to classify the pap smear microscopic image into normal, LSIL(Low Grade Intraepithelial lesion) and HSIL (High Grade Intraepithelial lesion).The proposed methodology concentrates on segmenting the cervical cells along with the removal of neutrophils which found to be greater hindrance for efficient classification of tissues. The proposed segmentation algorithm is highly generic that it could handle overlapped pap-smear cells to a better extent in comparison with other techniques. The morphology and texture based features are extracted from the segmented region of interest and optimized for accurate classification of cervical cells. Performance of classifiers and the significance of each feature have been analyzed from the classification results using machine learning techniques. Among the classifiers, Random Forest classifier provided an overall accuracy of $90 \%$ for cervical cancer screening using pap-smear microscopic images.
\end{abstract}

Keywords: HSIL, LSIL, Segmentation, Machine learning.

\section{Introduction}

Cytology is a well-established method for the detection of many diseases and which is safest and efficient technique. The most prominent outcome from cytology is that it has the capability to decrease the mortality and morbidity rate due to cervical cancer through the process of mass screening. A national screening was conducted in Taiwan from 1995 to 2006 and found that a $47.8 \%$ of decrease in invasive cancer incidence [1]. The first role of cytology is the early detection of dysplasia or pre-invasive cancer cells. When any abnormality is detected by cytology or any abnormal cell growth is found then the patient can be subjected to a biopsy examination and subsequent surgical treatment and according to that the further growth of the cancer cells can be stopped at an early stage [2]. Classical cytology is a microscopic diagnosis technique which observes the specialized cells and using the descriptive criteria it does a qualitative examination. This method is found to be inconsistent due to the subjective variability of independent observers. Advanced technologies including smear preparation, sampling, or screening quality control has been used for the reduction of false negative rate in screening [3-7]. Artificial intelligence technique by using computerized image 
analysis can be used for the detection and diagnosis of tumours or abnormal cell growth in cytopathology. The artificial method provides more accurate evaluation result of nuclear morphology.

Meng-Husiun Tsai et al [8] presented a study on cytoplasm and nucleus contour (CNC) detection to aid in the cervical cancer screening. They proposed a color differenced based method called Maximal color difference (MCD) to derive the contour of nucleus and cytoplasm. They adopted median filter to suppress the unwanted information present in the digitized microscopic smear image. The results indicates that the $\mathrm{CNC}$ detector fails to distinguish between two adjacent objects if they exhibits similar color.

Krishnan $\mathrm{N}$ et al [9] proposed a methodology for the segmentation of the cell nucleus of cervical cancer images which automatically extracts the region of interest and boundary of the object from the background. To extract the exact boundary of the corresponding object they used two parameters namely, gradient magnitude and directional information. The observed results indicates that the method fails to segment the overlapped cells.

Asli Kale et al [10] proposed a two phase method for the segmentation of cells in Pap smear test images. In the first phase the images are segmented by using a non-parametric hierarchical segmentation algorithm based on spectral shape information as well as the gradient information. In the second phase the segmented images are classified to obtain nucleus regions and areas of the cytoplasm. The proposed method performed well for pap smear test images having only a single cell and the images containing many overlapping cells.

Marina E. Plissiti et al [11] proposed a fully automated method which employs watershed transform to detect boundaries of the cell nuclei in conventional Pap smear images. The performance analysis of this technique was done based on the identification of the position of the nuclei. The watershed method was compared with the segmentation results of the gradient vector flow (GVF) deformable model and found that watershed transform technique captures the boundaries of the nuclei more accurately the than the other model and which leads to much improved performance of the clustering algorithm.

Youyi Song et al [12] identified a methodology of multiscale convolutional network(MSCN) and graph-partitioning-based method for segmentation of cervical cytoplasm and nuclei accurately. To localize the appearance of distinctive boundary for splitting the touching nuclei this method learned the parameters of the target object such as texture, shape, and contextual information. The results from this analysis showed that proposed method gave much better results and outperforms the existing methods.

Edwin Jayasingh Mariarputham et al [13] presented a nominated texture based cervical cancer (NTCC) classification system for the classification of the seven categories of the classes of the Pap smear images. By using this method there are seven sets of texture features which includes totally 24 features were extracted and the classification of the images are performed by using a few types of support vector machine (SVM) and neural network (NN) classifiers.

In this work novel methodology for segmentation \& feature extraction is proposed for abnormality analysis of cervical cancer. This methodology achieves an efficiency of $90 \%$ for cancer detection.

\section{Methodology}

\section{Acquisition of Pap-smear Images}


A high-resolution digital camera (Micropublisher 3.3) is used to capture cytological images and it is mounted on a microscope (Olympus BX 53) and the images are stored in a digital format. Three different scales of magnification (100x, 400x, and 1000x) are used to capture the cell images. It captured totally 3000 cell images from 15 pap-smear slides which includes both normal and abnormal cases. Images used in this method has a display resolution of $1024 \times 768$ pixels having 24 bit depth.

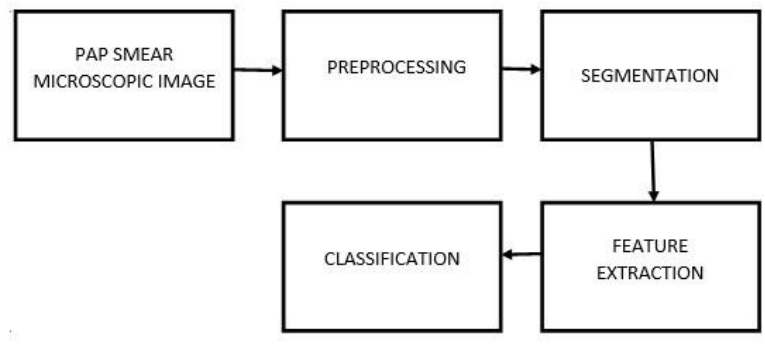

\section{Block Diagram of Proposed Methodology}

\section{Pre-processing and Segmentation}

The automated screening of cervical pathology is highly based on the characteristics of the pap smear microscopic image. The fundamental need in any automated image based diagnostic system is segmenting the region of interest, in case of pap smear microscopic image the main objective is to segment the cells present in the image. The common problem experienced and observed in the existing methods in the object extraction from the pap smear image is due to the overlapping nature of cells, artefacts created because of infections of the cervical tissues and also the staining procedure and dyes used for staining. The proposed algorithm attempts to overcome these drawbacks by means of employing proper preprocessing, segmentation, and classification based on novel features extracted from the segmented region of interest from the pap smear microscopic image.

The input microscopic images of pap-smear slide are pre-processed in order to enhance the image quality followed by segmentation algorithms performed on the same. The preprocessing of image is done in such a way that that it helps in better segmentation. Local contrast enhancement is done along with bilateral filtering with spatial parameter equal to 3 and range parameter equal to 50. .Segmentation process subdivides an image into different regions that are homogenous in some image characteristics. Various techniques can be considered as criteria for segmentation namely color, edges, and texture.The segmentation algorithm is aimed for the detection of nuclei locations and the nuclei boundary delineation with respect to the digitized conventional pap-smeared images.

In real time, most of the pap-smear images were found to consist of inflammatory infiltrations by neutrophils. The presence of neutrophils in turn reduces the information of the cervical cells lying underneath and hence the neutrophils need to be removed before application of any segmentation algorithm. The segmentation algorithm based on phansalkar local thresholding with a radius parameter of 13.5 which is set according to the efficiency of segmenting the input image. The local thresholding is followed by morphological operation dialation. This algorithm segments the nuclei along with the neutrophils. The segmented neutrophils and nuclei were separated based on their corresponding features. 


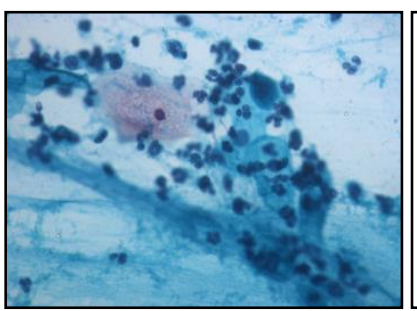

(a) Input original image
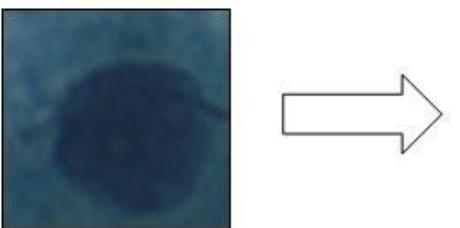

(b) Segmented Object

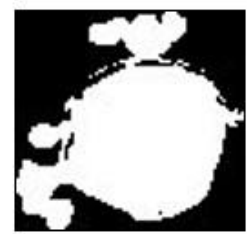

Object Extraction from the Segmented Images

Since most of the pap-smear images consist of inflammatory infiltration by neutrophils, extraction of neutrophils was found to be of high significance. The proposed segmentation algorithm effectively segments almost all the nuclei and neutrophils in an image. The overlapping of nucleus and netrophils exhibits challenges in the segmentation process. The main characteristics that discriminate between nucleus and netrophils are the texture and morphology ,which were considered as important features in the classification of segmented region of interest from the pap smear microscopic image as artefacts and nucleus in the proposed algorithm.

\section{Feature Extraction}

Generation of significant descriptors having good discriminative ability is required for the efficient separation of the true nuclei from the total segmented regions. The variations of cell structure and the extent of the cell spread in the tissue have to accurately captured to effect accurate computer aided diagnosis of cancer. The extraction of these features enumerates these changes. The extraction of features provides information of two types in the image, :(i) spatial arrangements of the pixels, (ii) intensity values of the pixels. The main focus of feature extraction in pap image lies in the arrangement of the chromatin within the cell nuclei. This is due to the fact that the eu- and hetero-chromatin serves as a significant determinant for different states of malignancy. For this reason, it is important to describe the properties of the chromatin. There are two approaches to describing the same, namely; (i) structure approach and (ii) texture approach.

\section{Shape Features}

The Identified region of interest of the boundaries of the nuclei are expected to exhibit elliptical form. Several features are examined to illustrate this characteristic. An important feature in the classification of cells and debris is the shape of the region. There are a large number of techniques available for shape description [14]. The shape features which are to be 
extracted from nucleus and cytoplasm of the cervical cell are categorized into two sets as nucleus related features and cytoplasm related features. Derived features in addition to the shape features are also included. Nucleus related features are Area, Centroid, Eccentricity, Equivalent Diameter, Major Axis Length, Minor Axis Length, Position, Roundness, Perimeter and Brightness. Nucleus cytoplasm ratio and cytoplasm related features are Area, Centroid, Eccentricity, Equivalent Diameter, Major Axis Length, Minor Axis Length, Perimeter, Brightness and Roundness. Since the region of study is only nuclei, features are extracted from only nuclei. In the detected boundary region the six main features were extracted namely; axis length, Circularity, circle with equivalent diameter and equivalent area as the region, Eccentricity, Major \& minor axis length of the detected region. Based on the morphological characters of the segmented regions from a histopathological image, following features have been extracted. Inputs from the expertise, the following three main distinguishing features were considered.

\section{Shape}

Shape of the malignance cancer is the numerical value of the nucleus parameters. So the presence of the malignance cancer is consider as one of the important feature.

\section{Nucleus area}

In general, the identification a high nuclei area value is show that the presence of abnormal cells. From the segmented nuclei/ neutrophil, the counting of neighbouring pixels is used to evaluate the nucleus area. Area of the nuclei and neutrophils are in square pixels in nature. Sometime its nature in calibrated square.

\section{Nucleus longest diameter}

The circle covered longer diameter of nuclei/neutrophils is knows as nucleus longest diameter. On the objects border, distance between to pixels forms a line. The length of this line is calculated.

\section{Nucleus shortest diameter}

The circle covered the shortest diameter a nuclei/neutrophils is denoted by nucleus shortest diameter. From this circle distance between pixels on the borders form a line, and the nucleus shortest diameter is evaluated from this line.

\section{Nucleus elongation}

The ratio of the shortest and longest diameter of the nucleus object is known as elongation, which is denoted by

Nelong $=$ Nshort diameter/ Nlong diameter

\section{Nucleus roundness}

The ratio of actual area and the area of nucleus longest diameter is called as nucleus roundness. And it is denoted by Nroundness

$$
\begin{aligned}
& \mathrm{N}_{\text {circle }}=\left((\mathrm{pi} / 4) * \mathrm{~N}_{2} \text { long }\right) \rightarrow \mathrm{N}_{\text {roundness }}=\mathrm{N}_{\text {area }} / \mathrm{N}_{\text {circle }} \\
& \text { Roundness is the inverse of Aspect Ratio given by } \\
& (4 \times[\text { Area }]) /(\pi \times[\text { Majoraxis }] 2)
\end{aligned}
$$

\section{Nucleus perimeter}

The perimeter length around the nuclei / neutrophils is known as Nucleus perimeter.

Nucleus position

The formation of nucleus in the center of the cytoplasm is also known as nucleus position. This position is calculated from the centricity value of nucleus and the cytoplasm, and it is represented as Npos

$$
\mathrm{N}_{\mathrm{pos}}=\{2 \mathrm{a} *((\mathrm{xN}-\mathrm{xC}) 2+(\mathrm{yN}-\mathrm{yC}) 2) 1 / 2\} / \mathrm{Clong}
$$


Where, the position is evaluated from the centricity of $(\mathrm{xN} ; \mathrm{yN})(\mathrm{N}-\mathrm{Nucleus})$ and $(\mathrm{xC}$; yC) (C-Cytoplasm).

\section{Centroid}

The center point of the nuclei/ neutrophils is used to calculate the centroid. For this, the average value of all the pixels coordinates in the image are utilized.

\section{Bounding rectangle}

The smallest rectangle enclosing the nuclei/ neutrophils.

\section{Circularity}

The circularity is defined as a ratio between area and square of perimeter given by $4 \pi \times$ [Area]/[Perimeter] 2 with a value of 1.0 indicating a perfect circle.

\section{AR (Aspect Ratio)}

The particle's fitted ellipse aspect ratio is denoted by [MajorAxis]/[MinorAxis]

\section{Solidity}

The ratio between total are and convex area is simply called as solidity, and which is denoted by $[$ Area $] /[$ Convexarea $]$

\section{Feret, FeretAngle, MinFeret, Feret $X$, FeretY}

In the selection boundary, the distance between any two points is measured and the longest distance is considered, and it is also represented as maximum calliper. The Feret's diameter angle ranges between $0 \mathrm{o}$ to $180 \mathrm{o}$ is called as FeretAngle.

\section{IntDen (Integrated Density), RawIntDen (Raw- Integrated Density)}

In the segmented region the pixels sum value is equivalent to the product of Area and also equivalent to mean gray value. The sum of these pixel values are evaluated from raw integrated density

\section{Color Intensity}

The distribution of colour pixel in the selected region is represents the colour intensity of that particular region of a cell. When the nuclei area is increased then the presence of pixels in the cell darkness also increased. In general, the colour intensity distribution between cancerous and non-cancerous cells probably always different in nature. Based on the cancer cell intensity, cancerous calls are classified in to Low grade and High grade. Low-grade cells are fall under circular in nature whereas high grade cells are mostly in tapered and spindle in nature.

\section{a) Nucleus Brightness}

With the help of average apparent brightness of segmented nucleus colours wavelength, nucleus brightness is evaluated. The nucleus brightness is denoted as

$$
\mathrm{Y}=0.299 * \operatorname{Red}^{1}+0.587 * \mathrm{Green}^{1}+0.114 * \mathrm{Blu}^{1}
$$

Where Red ${ }^{1}$, Green $^{1}$ and Bluelis the average intensity value for wavelength of each colours. They are weighted by the perceived brightness of the human eye.

\section{b) Nucleus Maxima/Minima} radius.

The minimum and maximum gray values within the nuclei/ neutrophils inside of a 3 pixel

\section{c) Centre of mass}

The brightness average value of the $\mathrm{x}$ and $\mathrm{y}$ coordinates of all pixels present in the image is replaced or weighted is utilized for evaluated the center of mass. The first order spatial moments are represented by these weighted coordinates.

\section{d) Skew}

The mean value of the third order spatial moment is known as skew.

e) Kurtosis

The mean value of the fourth order spatial moment is represent as kurtosis. 


\section{Textural Features}

An essential characteristics of entities is also known as texture. Few important textures in the images are brightness, colour, scale, shape, etc. The first and higher order statistical features are extracted from the histogram in order to analyze the texture features in the proposed methodology.Local binary patterns are consider as a texture descriptor and these descriptors were calculated. Thus, for every segmented region Entropy, Energy, Correlation co-efficient have been determined. Texture features to be are extracted using Gray Level Cooccurrence Matrix (GLCM) [14]. Texture based features that can be used for classification of Pap-smear tissues are as follow: Contrast, Correlation, Difference entropy, Difference variance, Entropy, Homogeneity, Inverse difference moment, Information measure I, Information measure II, Maximal correlation coefficient, Sum average, Sum entropy, Sum variance, and Variance. Out of the features mentioned, the texture features extracted for classification of pap-smear images includes Correlation, Entropy, Energy/ Angular second moment ,Inverse Difference Moment and Contrast.

\section{Energy}

The second order moment of angular and the uniformity is also known as energy. Uniformity is nothing but a measurement of textural uniformity of a selected image. The square sum of individual pixel, returns the grayscale distribution homogeneity of images and texture crudeness. In the co-occurrence matrix, the similar data may gave some small energy profile. At the same time the unequal data in the co-occurrence matrix gave a high energy profile. Once the gray level distribution value is periodic nature or in constant, then the energy reaches its highest value. In a homogenous image, large value of energy information is based on the few dominant gray tone transitions. However, from the normalized GLCM, sum of the squared element is represented as energy

$$
\begin{aligned}
& \text { En }_{\mathrm{d}, \theta}=\sum_{\mathrm{i}=1} \sum_{\mathrm{j}=1} \mathrm{p}_{\mathrm{d}, \theta}(\mathrm{i}, \mathrm{j})^{2} \\
& \text { Correlation }
\end{aligned}
$$

\section{Correlation}

The connection between the pixel and neighbouring pixel distribution throughout the entire image is denoted as correlation. In this process, correlation between each attributes and the labelled attributes are calculated. And absolute or square is taken for these calculated values and considered it as its weight. The image texture consistency is evaluated from this correlation value, which is denoted by.

Correlation $_{\mathrm{d}, \theta}=\left(\sum_{\mathrm{i}=1} \sum_{\mathrm{j}=1}(\mathrm{i}-\mu \mathrm{x})(\mathrm{j}-\mu \mathrm{y}) \mathrm{p}_{\mathrm{d}, \theta}(\mathrm{i}, \mathrm{j})\right) /(\sigma \mathrm{x} \sigma \mathrm{y})(4.7)$

in which $\mu \mathrm{x}, \mu \mathrm{y}, \sigma \mathrm{x}, \sigma \mathrm{y}$ and were described as follows:

$$
\begin{aligned}
& \mu_{\mathrm{x}}=\sum_{\mathrm{i}=1}{ }^{\mathrm{Ng}} \sum_{\mathrm{j}=1}{ }^{N g} \mathrm{ip}(\mathrm{i}, \mathrm{j}) \\
& \mu_{\mathrm{y}}=\sum_{\mathrm{i}=1}{ }^{\mathrm{Ng}} \sum_{\mathrm{j}=1}{ }^{N g} \mathrm{jp}(\mathrm{i}, \mathrm{j}) \\
& \sigma_{\mathrm{x}}=\left(\sum_{\mathrm{i}=1}{ }^{\mathrm{Ng}} \sum_{\mathrm{j}=1}{ }^{\mathrm{Ng}}\left(\mathrm{i}-\mu_{\mathrm{x}}\right)^{2} \mathrm{p}(\mathrm{i}, \mathrm{j})\right)^{1 / 2} \\
& \sigma_{\mathrm{y}}=\left(\sum_{\mathrm{i}=1}{ }^{\mathrm{Ng}} \sum_{\mathrm{j}=1}{ }^{\mathrm{Ng}}\left(\mathrm{i}-\mu_{\mathrm{y}}\right)^{2} \mathrm{p}(\mathrm{i}, \mathrm{j})\right)^{1 / 2} \\
& \text { Entropy }
\end{aligned}
$$

In the image texture, the complexity and non-uniformity is utilized to represents the entropy of that selected image region

$$
\text { Entropyd, } \theta=-\sum \mathrm{i}=1 \sum \mathrm{j}=1 \mathrm{pd}, \theta(\mathrm{i}, \mathrm{j}) \log (\mathrm{pd}, \theta(\mathrm{i}, \mathrm{j}))
$$

\section{Contrast}

Image sharpness and the texture depth grooves are evaluated from the contrast. Higher value of sharpness and contrast is directly proportional to the deeper of texture grooves. At the same time, lower amount of contrast may leads to shallow texture grooves. These lower contrast blurred images also. The large number of pixels with high difference in grayscale (e.g., contrast profile) is associated with higher values of contrast

$$
\text { Contrast }_{\mathrm{d}, \theta}=\sum_{\mathrm{i}=1} \sum_{\mathrm{j}=1}(\mathrm{i}-\mathrm{j})^{2} \mathrm{p}_{\mathrm{d}, \theta}(\mathrm{i}, \mathrm{j})
$$




\section{Novel colour based nucleus cytoplasm ratio features}

There are three features which are framed in order to estimate the nucleus cytoplasm ratio. The nucleus cytoplasm ratio is one of the important feature which can differentiate a cancerous and a normal cell. But finding it out is difficult because it requires segmentation of both nucleus and cytoplasm. The segmentation of nucleus can be done effectively but cytoplasm segmentation is a difficult task as it involves complexities like overlapping cells, staining artifacts, improper staining and irregular shape of cells. Instead of segmenting the cytoplasm the proposed features utilize the colour surrounding the well segmented nucleus inside a fixed radius. This colour information inside the fixed radius around the nucleus can give indirect information about the nucleus cytoplasm ratio.

Red Cytonucleus

This feature measures the mean of red component of the RGB image pixels inside the fixed radius

$$
\mathrm{RC}=\left(\sum \mathrm{i}=\mathrm{n}(\mathrm{Ri},)\right) / \mathrm{n}
$$

Blue Cytonucleus

This feature measures the mean of blue component of the RGB image pixels inside the fixed radius

$\mathrm{BC}=\left(\sum \mathrm{i}=\mathrm{n}(\mathrm{Bi})\right) / \mathrm{n}$

Green Cytonucleus

In This feature measures the mean of green component of the RGB image pixels inside the fixed radius

$$
\mathrm{GC}=\left(\sum \mathrm{i}=\mathrm{n}(\mathrm{Gi},)\right) / \mathrm{n}
$$

Thus, for the taxonomy of pap-smear images, 42 shape-based, textural-based and colour based features extracted includes Area, Mean, Median, Mode, Standard Deviation, Minimum, Maximum, \%Area, X, Y, XM, YM, Perimeter, BX, BY, Width, Height, Major, Minor, Angle, Circularity, Feret, IntDen, RawIntDen, FeretX, FeretY, FeretAngle, MinFeret, AR, Round, Solidity, Skew, and Kurtosis, Angular second moment, Energy, Entropy, Correlation coefficient, Contrast Inverse Difference Moment, Red cytonucleus, Green cytonucleus, and Blue cytonucleus have been found to efficiently differentiate between normal and abnormal cells

\section{Classification}

The cervical screening systems are utilised to determine the presence of precancerous lesions. The classification can be performed in either of the two ways, determination of a cell as normal or abnormal, or assigning it one of various levels of dysplasia. As it is crucial to determine the severity of cancer, classification algorithm concentrates on determining a cell to be normal or abnormal. The most important features for classification are nucleus and cytoplasm area, red cytonucleus, nucleus and cytoplasm brightness/minima/maxima/ and nucleus roundness.

In the first stage of classification,the proposed methodology eliminate the nutrophils from the nucleus. The first stage has only two classes which are nucleus and nutrophils and it is trained using random forest classifier. The samples which are classified as nucleus in the first 
stage are taken for second stage of classification where they are classified as either normal, LSIL or HSIL.

Classification of pap-smear images involves two steps: Training the system and testing the performance of the trained system. The performance of a system depends on the classifier selected and the optimized features. A trainer set of 319 data have been used to train the system with RandomForest classifier with features extracted from the images as attributes. Initially 39 attributes including the texture-based ,colour-based and shape-based features have been included for classification which was later optimized to give higher \% accuracy when supplied with a test set. The classifier was trained with 10 -fold cross validation which resulted in an accuracy percentage of $94.5 \%$ for stage 1 and $90 \%$ accuracy for stage 2 overall.

\begin{tabular}{|l|l|l|l|l|}
\hline Classification & Model & $\begin{array}{l}\text { Overall } \\
\text { Accuracy }\end{array}$ & $\begin{array}{l}\text { Nutrophils } \\
\text { class } \\
\text { Accuracy }\end{array}$ & $\begin{array}{l}\text { Nucleus } \\
\text { class } \\
\text { Accuracy }\end{array}$ \\
\hline $\begin{array}{l}\text { Nutrophils vs } \\
\text { nucleus }\end{array}$ & $\begin{array}{l}\text { Random } \\
\text { Forest }\end{array}$ & $94.5 \%$ & $100 \%$ & $94.5 \%$ \\
\hline
\end{tabular}

\begin{tabular}{|l|l|l|l|l|l|}
\hline Classifier & Model & $\begin{array}{l}\text { Overall } \\
\text { Accuracy }\end{array}$ & $\begin{array}{l}\text { Normal } \\
\text { class } \\
\text { Accuracy }\end{array}$ & $\begin{array}{l}\text { HSIL } \\
\text { class } \\
\text { Accuracy }\end{array}$ & $\begin{array}{l}\text { LSIL } \\
\text { class } \\
\text { Accuracy }\end{array}$ \\
\hline $\begin{array}{l}\text { Normal } \\
\text { vs HSIL } \\
\text { vs LSIL }\end{array}$ & $\begin{array}{l}\text { Random } \\
\text { Forest }\end{array}$ & $90 \%$ & $93.75 \%$ & $90 \%$ & $100 \%$ \\
\hline $\begin{array}{l}\text { Normal } \\
\text { vs HSIL } \\
\text { vs LSIL }\end{array}$ & $\begin{array}{l}\text { Gradient } \\
\text { Boosting }\end{array}$ & $80 \%$ & $80 \%$ & $83.30 \%$ & $75 \%$ \\
\hline
\end{tabular}

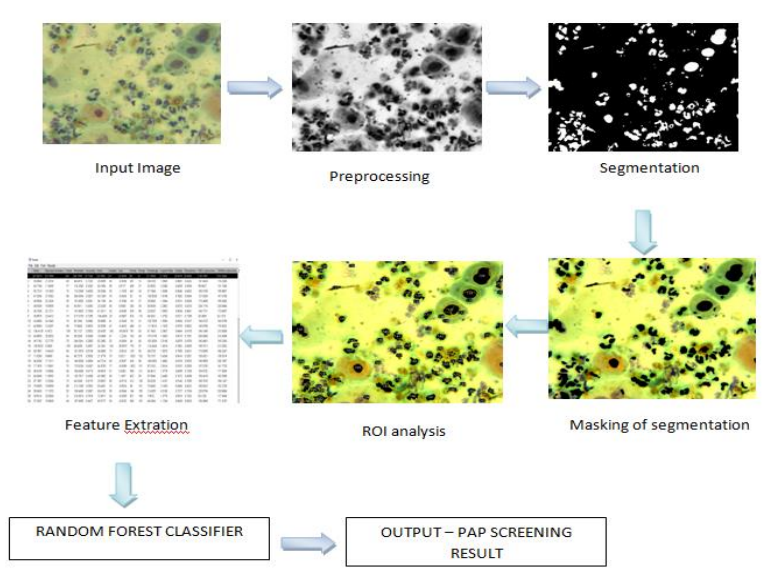

\section{Conclusion and Future Work}

The objective of this work is to develop an image processing algorithm for a complete analysis of cervical cancer using pap-smear images. Though various segmentation algorithms 
were tried, the problem of clustering and over-lapping of cell structures makes the segmentation algorithms less efficient as the pixel intensity and other variations among the ROI and background are obscured. A new methodology is proposed for abnormality analysis. Prior to analysis, the images are pre-processed using bilateral filtering technique and contrast enhanced for better image segmentation. The proposed segmentation algorithm was so generic that it was able to process images containing a single cell as well as overlapping cells to a comparable extent. With the proposed image processing algorithm, extracted shape-based and texture-based features and the performance of Random Forest classifier, an efficiency of $86.6 \%$ have been achieved for cancer analysis. The accuracy \% of this methodology can be improved by further enhancing the segmentation algorithm. This novel methodology is better than various other existing methodologies. Furthermore, scope for future work is to increase the accuracy percentage above $90 \%$. In many cases, the shape and size of the normal nuclei match with that of the abnormal ones (LSIL and HSIL). This, unavoidably, results in misclassification of normal, LSIL, HSIL nuclei and neutrophils. Most importantly, as there are myriad of inter-class and intra-class variations among the cervical cell types in the pap images, all algorithms fail to segment the object of interest (nucleus) accurately. These image-oriented difficulties can be handled by working the algorithm for much larger dataset with expert annotations, whereby deep machine learning of the images by the algorithm will result in higher classification accuracy.

\section{References}

[1] Y-Y Chen,1 S-L You,2 C-A Chen,3 L-Y Shih,4 S-L Koong,4 K-Y Chao,4 M-L Hsiao,4 C-Y Hsieh,3 and C-J Chen1,2, ,Effectiveness of national cervical cancer screening programme in Taiwan: 12-year experiences, Br J Cancer. 2009 Jul 7; 101(1): 174-177.

[2] Adam E, Kaufman RH, Adler-Storthz K, et al. A prospective study of association of herpes simplex virus and human papillomavirus infection with cervical neoplasia in women exposed to diethylstilbestrol in utero. Int J Cancer. 1985; 35(1):19-26.

[3] Tolles WE, Bostrom RC. Automatic screening of cytological smears for cancer: the instrumentation. Ann N Y Acad Sci.1956;63(6):1211-8.

[4] Spencer CC, Bostrom RC. Performance of the cytoanalyzer in recent clinical trials. J Natl Cancer Inst. 1962;29:267-76.

[5] BD.Focal Point; 2014. http:// www.bd.com/ tripath/ labs/ fpscreening. asp.

[6] Kardos TF. The FocalPoint system. Cancer Cytopathol. 2004;102(6):334-9.

[7] Ruhul QM, et al. Does the ThinPrep imaging system increase the detection of high-risk HPVpositive ASC-US and AGUS? The Women and Infants Hospital experience with over 200,000 cervical cytology cases. Cytojournal. 2009;6(1):15

[8] Meng-Husiun Tsai,, Yung-Kuan Chan,, Zhe-Zheng Lin,, Shys-Fan Yang-Mao,, Po-Chi Huang Nucleus and cytoplast contour detector of cervical smear image, Journal Pattern Recognition Letters archive Volume 29 Issue 9, July, 2008,Pages 1441-1453

[9] N. Krishnan \& S. Naga Nandini Sujatha,'Segmentation of Medical Images Using Directional Force Active Contour Models' IJACE International Journal of Advanced Computer Engineering • JulyDecember $2011 \cdot$ Volume $4 \cdot$ Issue 2.

[10] Asli Kale ,'Segmentation and Classification of Cervical Cell Images a thesis submitted to the department of computer engineering and the institute of engineering and science of bilkent university in partial fulfillment of the requirements for the degree of master of science By January, 2010.

[11] Marina E. Plissiti, Christophoros Nikou , Member, IEEE , and Antonia Charchanti,Automated Detection of Cell Nuclei in Pap Smear Images Using Morphological Reconstruction and Clustering, IEEE TRANSACTIONS ON INFORMATION TECHNOLOGY IN BIOMEDICINE, VOL. 15, NO. 2, MARCH 2011233 
[12] Y. Song, L. Zhang, S. Chen, D. Ni, B. Lei, and T. Wang, Accurate Segmentation of Cervical Cytoplasm and Nuclei Based on Multi -scale Convolutional Network and Graph Partitioning,IEEE Trans. Biomed. Eng ., 2015

[13] Edwin Jayasingh Mariarputham 1,* and Allwin Stephen,Nominated Texture Based Cervical Cancer Classification, Comput Math Methods Med. 2015.

[14] Amutha devi, Gayathri Monicka.J, "Development of fuzzy approach to predict the fetus safety and growth using AFI” Journal of super computing” Dec-2019 DOI 10.1007/s11227-019-03099-8

[15] Dr.Gayathri Monicka, Apurva,Nishant and Piorebello "Design and Development of a Smart Electric Trike with detachable handle for Differently Abled" Elsevier material today . https://doi.org/10.1016/j.matpr.2020.06.261.

[16] D. Zhang, G. Lu, Review of shape representation and description techniques, Pattern Recognition 37 (1) (2004) 1-19.

[17] Haralick RM, Shanmugam K, Dinstein IH. Textural features for image classification. IEEE Trans Syst Man Cybern.1973;6:610-21. 